\title{
Efecto de coberturas naturales en el almacenamiento en frío de frutos de palto (Persea americana Mill) cv. Hass
}

\author{
Effect of natural coatings in cold storage of fruits of avocado (Persea americana Mill) cv. Hass
}

\author{
Liliana Zambrano M. ${ }^{1}$; Guillermo J. Parodi M. ${ }^{2}$
}

\begin{abstract}
Resumen
El presente estudio se realizó para determinar el efecto de distintas coberturas naturales comestibles sobre la conservación poscosecha de frutos de palto cv. 'Hass'. Para ello, se utilizó como coberturas comestibles a los productos carboximetil celulosa, goma de tara, almidón de yuca y almidón de papa, en concentraciones de 0,2\%, 0,4\% y $0,6 \%$, las cuales se aplicaron a los frutos de palto cv. 'Hass' en poscosecha, previo a su almacenamiento en frío. Los frutos de palto se almacenaron por 30 días en una cámara de conservación en frío a $5{ }^{\circ} \mathrm{C}$ y con $85-90 \%$ de humedad relativa. El diseño estadístico utilizado fue el de completamente al azar con arreglo factorial de cuatro tipos de cobertura por tres concentraciones; se consideró, además, un tratamiento testigo como aditivo lineal. Todos los parámetros evaluados, a excepción del color externo, el pardeado de pulpa y la palatabilidad, fueron sometidos a una prueba de comparación de medias de Tuckey $(\mathrm{p} \leq 0,05)$. Los datos de color externo y pardeado de pulpa se analizaron mediante la prueba estadística no paramétrica de Kruskal-Wallis $(\mathrm{p} \leq 0,05)$ y la de palatabilidad a través de la prueba estadística no paramétrica de Friedman $(\mathrm{p} \leq 0,05)$. Los resultados obtenidos mostraron que los frutos evaluados a la salida de almacenaje tuvieron diferencias significativas respecto de la resistencia a la presión y porcentaje de materia seca, siendo esto más notorio en aquellos frutos que recibieron la cobertura de goma de tara al 0,6 \%. Asimismo, la evaluación de los frutos 14 días después de retirados del almacenamiento, mostró que aquellos que recibieron la cobertura de goma de tara al $0,6 \%$ tuvieron una menor pérdida de peso, un menor porcentaje de aceite, mayor resistencia a la presión y un menor cambio de color de la epidermis, no observándose pardeado de pulpa, logrando con ello una mayor aceptación por degustación. Palabras clave: almacenaje; coberturas comestibles; goma de tara; poscosecha.
\end{abstract}

Abstract

The present study was made to determine the effect of different natural edible coatings on postharvest conservation of avocado fruits cv. 'Hass'. Carboxymethyl cellulose, tara gum, mandioca starch and potato starch at concentrations of 0,2 $\%, 0,4 \%$ and $0,6 \%$, were applied to avocado fruits cv. 'Hass' before post-harvest storage. Fruits were stored at $5{ }^{\circ} \mathrm{C}$, with a relative humidity of $85-90 \%$, for 30 days. The statistical design used was ANOVA with a factorial arrangement of four types of coatings and three doses by coating, it was also considered a linear additive as control treatment. Tukey mean test $(\mathrm{p} \leq 0,05)$ was used. Evaluation of color skin and pulp browning were analyzed with non-parametric statistical tests of Kruskal-Wallis $(\mathrm{p} \leq 0,05)$ and tasting was analyzed with non-parametric statistical tests of Friedman $(\mathrm{p} \leq 0,05)$. Results showed that fruits evaluated at the end of storage had significant differences in pressure resistance and dry matter percentage; in fruits treated with tara gum coating of $0,6 \%$ these results were more evident. Evaluation of fruits at 14 days after the end of storage showed that the tara gum coating at $0,6 \%$ treatment allowed a lower weight loss and oil percentage, higher resistance to pressure and a lower change in skin color, without the presence of browning pulp and a better taste acceptance.

Keywords: storage; edible coating; tara gum; postharvest.

\section{Introducción}

El desarrollo de prácticas o procedimientos vinculados a mejorar y prolongar la vida útil de productos vegetales frescos ha sido y es un reto permanente para el logro de un adecuado manejo y conservación poscosecha de frutas y hortalizas (Pantastico, 1984). Para reducir rápidamente las funciones metabólicas de respiración y transpiración de los órganos vegetales una vez cosechados, se pueden utilizar una serie de procedimientos cuya aplicación se inicia desde el momento que estos son recolectados y dentro de los cuales destaca la aplicación del control de la temperatura; el preenfriamiento es uno de los primeros pasos que sigue la cadena de frío de un órgano vegetal en poscosecha (Wills et al., 1984). Sin embargo, el frío puede,

1 Bachiller en Agronomía. Universidad Nacional Agraria La Molina, Lima, Perú.

2 Ingeniero Agrónomo, Departamento de Horticultura, Facultad de Agronomía. Universidad Nacional Agraria La Molina, Lima, Perú. Email: gparodi@lamolina.edu.pe 
en muchos casos, generar alteraciones o daños que afectan la calidad final del producto durante el almacenaje por lo que el uso de coberturas, tanto en frutas como en otros órganos vegetales, puede permitir controlar la respiración y la senescencia, además de reducir parte de los efectos causados por el frío durante el almacenaje logrando, de esta manera, aminorar su deterioro (Baldwin, 1994). En el caso de frutos de palto cv. 'Fuerte' se ha encontrado que el uso de carbohidratos como el éster de sacarosa aplicados como cobertura comestibles al 1,2 \% actúa retardando la madurez y disminuyendo el desarrollo de desórdenes fisiológicos durante su almacenaje (Maibee, 1993). En algunos casos, la aplicación de estas coberturas ha logrado que se incrementen las concentraciones de dióxido de carbono en la porción interna de los frutos de palto, consiguiendo con ello reducir hasta en un 50 \% la pérdida de peso, además de lograr una reducción de la velocidad de maduración (Banks et al., 1997). Esto incluso ha contribuido a que no se presente una acelerada degradación de la clorofila en la epidermis de los frutos del palto cv. 'Hass' permitiendo mantener el color verde característico de una fruta no senescente (Maftoonazad y Ramaswamy, 2005). A su vez, el uso de coberturas a base de carnauba en palto cv. 'Hass', además de lograr reducir la deshidratación, también contribuyó a minimizar la sintomatología de pardeado externo e interno del fruto (Cancino, 2007).

El trabajo de investigación tuvo como objetivo estudiar el efecto de cuatro tipos de cobertura aplicadas en tres distintas concentraciones y evaluar su efecto en la conservación de frutos de palto (Persea americana Mill) cv. 'Hass', sometidos a almacenamiento en frío.

\section{Materiales y métodos}

Para el trabajo experimental se utilizaron frutos de palto cv. 'Hass' procedentes de un campo comercial de la empresa
JJC-Chilca. Los frutos presentaron a la cosecha un contenido mínimo de materia seca de 19,4\% correspondiente a un $9,2 \%$ de aceite en pulpa. Posteriormente, fueron trasladados al laboratorio de horticultura de la Universidad Nacional Agraria La Molina (UNALM) donde se lavaron y desinfestaron con una solución del producto comercial, DESFAN ${ }^{\circ}$ diluido al $1 \%$, para luego ser enjuagados en agua potable filtrada, secados con paños y luego recibir las coberturas a base de: carboximetil celulosa (CMC), goma de tara (GT), almidón de yuca (AY) y almidón de papa (AP) además de un testigo (T) el cual no recibió ninguna cobertura.

La aplicación de las coberturas se realizó mediante un rociadormanual.Paraasegurarlaadherenciadelascoberturas al fruto se utilizó PROFILM $®$ diluido al 0,01\%. Una vez concluida esta labor, los frutos se trasladaron a la empresa agroindustrial Torre Blanca (Huaral-Lima), lugar en el cual recibieron un tratamiento de preenfriamiento con aire forzado a $6{ }^{\circ} \mathrm{C}$ durante 10 horas, antes de su almacenaje definitivo en una cámara de frío graduada a la temperatura de $5{ }^{\circ} \mathrm{C}$ con $85-90 \%$ de humedad relativa, lugar en el que los frutos permanecieron por espacio de 30 días. Una vez concluida la fase de almacenamiento, los frutos se trasladaron a un ambiente con temperatura de $20-22{ }^{\circ} \mathrm{C}$ y $80-90 \%$ de humedad relativa donde estuvieron por 14 días con la finalidad de estimular su maduración organoléptica. En total, se consideraron para el presente trabajo de investigación 13 tratamientos (Tabla 1), cada uno formado por tres repeticiones y tres unidades experimentales (frutos) por repetición, haciendo un total de 232 frutos. El diseño experimental considerado fue el completamente al azar (DCA) dispuesto en arreglo factorial de cuatro coberturas (carboximetil celulosa, goma de tara, almidón de yuca y almidón de papa) por tres concentraciones $(0,2 \%, 0,4 \%$ y $0,6 \%)$, además de considerar un tratamiento aditivo lineal (testigo sin aplicación). Todos

Tabla 1. Descripción de los tratamientos aplicados en los frutos de palto (Persea americana Mill) cv. 'Hass' antes de su almacenaje en frío

\begin{tabular}{ccc}
\hline Tratamientos & Abreviaturas & Coberturas \\
\hline $\mathbf{1}$ & CMC 0,02\% & Carboximetil celulosa $0,2 \%+$ adherente $0,01 \%$ \\
$\mathbf{2}$ & CMC 0,4\% & Carboximetil celulosa $0,4 \%+$ adherente $0,01 \%$ \\
$\mathbf{3}$ & CMC 0,6\% & Carboximetil celulosa $0,6 \%+$ adherente $0,01 \%$ \\
$\mathbf{4}$ & $G T 02 \%$ & Goma de tara $0,2 \%+$ adherente $0,01 \%$ \\
$\mathbf{5}$ & $G T 0,4 \%$ & Goma de tara $0,4 \%+$ adherente $0,01 \%$ \\
$\mathbf{6}$ & $G T 0,6 \%$ & Goma de tara $0,6 \%+$ adherente $0,01 \%$ \\
$\mathbf{7}$ & $A Y 0,2 \%$ & Almidón de yuca $0,2 \%+$ adherente $0,01 \%$ \\
$\mathbf{8}$ & $A Y 0,4 \%$ & Almidón de yuca $0,4 \%+$ adherente $0,01 \%$ \\
$\mathbf{9}$ & $A Y 0,6 \%$ & Almidón de yuca $0,6 \%+$ adherente $0,01 \%$ \\
$\mathbf{1 0}$ & $A P 0,2 \%$ & Almidón de papa $0,2 \%+$ adherente $0,01 \%$ \\
$\mathbf{1 1}$ & $A P 0,4 \%$ & Almidón de papa $0,4 \%+$ adherente $0,01 \%$ \\
$\mathbf{1 2}$ & $A P 0,6 \%$ & Almidón de papa $0,6 \%+$ adherente $0,01 \%$ \\
$\mathbf{1 3}$ & $T$ & Tratamiento testigo “0” \\
\hline
\end{tabular}


los parámetros, a excepción del color externo, pardeado de pulpa y palatabilidad se sometieron a una evaluación de comparación de medias de Tukey $(\mathrm{p} \leq 0,05)$. En el caso del color externo como del pardeado de pulpa, se usó la prueba estadística no paramétrica de Kruskall - Wallis ( $p$ $\leq 0,05)$ (Corzo, 2005) y para el análisis de palatabilidad se utilizó la prueba estadística no paramétrica de Friedman ( $\mathrm{p}$ $\leq, 05)$ (Corzo, 2005). Los tratamientos se evaluaron tanto al concluir el período de almacenaje de los frutos como a los 14 días después de concluido el almacenamiento en frío; a excepción de la evaluación del cambio de color externo el cual se realizó a los $0,4,8$ y 14 días después de concluido el período de almacenamiento en frío, mientras que la prueba de palatabilidad se realizó solamente a los 14 días después de concluido ese período. Los parámetros evaluados fueron:

\section{Pruebas Físicas}

- Pérdida de Peso: Se determinó mediante una balanza marca "AE" de $\pm 0,1 \mathrm{~g}$ de precisión.

- Color externo: Se utilizó la Tabla 2. La cual se elaboró a partir de la escala de grados de color externo propuesta por Parodi y Lerner (1995) y que se comparó con la cartilla de color para tejidos vegetales (FAN 4 y FAN 3) de la RHS (2001). Para la calificación del grado de color de la epidermis del fruto, se consideró como premisa que más del $50 \%$ del color de la piel en el fruto evaluado debía encontrarse en alguna de las categorías indicadas en la mencionada Tabla.

Tabla 2. Categorías y grados para la evaluación del color externo en frutos de palto (Persea americana Mill) cv. 'Hass'

\begin{tabular}{lc}
\hline \multicolumn{1}{c}{ Categorías } & Grado \\
\hline 137 A-D o 138 A (Green Group) & $\mathbf{1}$ \\
$\begin{array}{l}\text { 146 A o 148 A (Yellow Green } \\
\text { Group) }\end{array}$ & $\mathbf{2}$ \\
$\begin{array}{l}\text { 197 A o 199 A (Greyed Green - } \\
\text { Group Grey Group) }\end{array}$ & $\mathbf{3}$ \\
200 B o 200 C (Brown Group) & $\mathbf{4}$ \\
200 A (Brown Group) & $\mathbf{5}$ \\
\hline
\end{tabular}

- Determinación de la dureza: Se utilizó un penetrómetro digital TR Turoni Modelo 53205 con vástago de 5/16". La medida se realizó en la porción central del fruto tomándose dos datos por fruto, cada uno en extremos opuestos. Las lecturas fueron registradas en kilogramos-fuerza (kg-f).

- Pardeado de pulpa: Se evaluó el nivel de oscurecimiento de la pulpa proponiéndose una escala hedónica cuyos grados y categorías se indican en la Tabla 3.

- Materia seca de la pulpa del fruto: Se colocaron $50 \mathrm{~g}$ de pulpa fresca de palto en una estufa durante un mínimo de 24 horas a $70{ }^{\circ} \mathrm{C}$. Luego se pesó en una balanza digital marca "AE" de $\pm 0,1 \mathrm{~g}$ de precisión y se estableció el valor final para luego transformarlo en porcentaje.
Tabla 3. Descripción de los grados de evaluación de pardeado de pulpa del fruto del palto (Persea americana Mill) cv. 'Hass'

\begin{tabular}{lc}
\hline \multicolumn{1}{c}{ Categorías } & Grado \\
\hline $\begin{array}{l}\text { No existe pardeado de la pulpa } \\
\text { Existe un pardeado de pulpa mayor a } 0,1 \%\end{array}$ & 1 \\
y menor a $5 \%$ & 2 \\
$\begin{array}{l}\text { Existe pardeado de pulpa mayor a } 5 \% \text { y } \\
\text { menor a } 15 \%\end{array}$ & 3 \\
Existe pardeado de pulpa mayor a $15 \%$ & 4 \\
\hline
\end{tabular}

\section{Pruebas químicas}

- Determinación de porcentaje de aceites: Se utilizaron 2,5 g de materia seca de pulpa de palto por análisis, extrayéndose el total de aceite mediante éter de petróleo con un equipo extractor Soxhlet, según procedimiento de la Association of Official Analytical Chemist International (AOAC, 1990).

\section{Prueba sensorial}

- Palatabilidad: Se realizó sólo a los 14 días después de concluido el periodo de almacenamiento en frío. Para la calificación se trabajó con un panel degustador de ocho personas (Cancino, 2007), a las cuales se pre entrenó antes del proceso de degustación de las muestras de palta. Asimismo, para la calificación del nivel de palatabilidad de la fruta, se utilizó una escala hedónica propuesta por Lizana e Irrazabal, citados por Parodi y Lerner (1995), cuyos grados y categorías se indican en la Tabla 4.

Tabla 4. Grados de evaluación de la palatabilidad del fruto del palto (Persea americana Mill) cv. 'Hass'

\begin{tabular}{lc}
\hline \multicolumn{1}{c}{ Categorías } & Grado \\
\hline Me disgusta extremadamente & 1 \\
Me disgusta mucho & 2 \\
Me disgusta medianamente & 3 \\
Me disgusta algo & 4 \\
Me es indiferente & 5 \\
Me gusta algo & 6 \\
Me gusta medianamente & 7 \\
Me gusta mucho & 8 \\
Me gusta extremadamente & 9 \\
\hline
\end{tabular}

\section{Resultados y discusión}

\section{Pérdida de Peso}

El análisis estadístico correspondiente presentó diferencias estadísticas a nivel de la interacción de coberturasconcentración, no se observaron diferencias significativas en la pérdida de peso cuando se evaluaron las diferentes coberturas para cada una de las concentraciones empleadas (Fig. 1); pero sí se presentaron diferencias estadísticas en cada una de las coberturas en función de la concentración empleada; es así que las coberturas de GT al 0,6 \% y CMC al 0,2 \% reportaron un mejor efecto logrando reducir ligeramente la pérdida de peso (Fig. 2). A los 14 
días después de concluido el período de almacenaje en frío, no se obtuvieron diferencias significativas en ningún tratamiento. A pesar de que no se pudo observar un marcado efecto de las coberturas empleadas sobre la reducción de la pérdida de peso en el palto cv. 'Hass', sí es importante indicar que, dependiendo de la concentración empleada en algunas de las coberturas, se pudo conseguir reducir la pérdida de peso, aspecto que coincide con lo mencionado por Banks et al. (1997), quienes observaron también una menor pérdida de peso en los frutos de palto cv. 'Hass' dependiendo del tipo de cobertura y concentración usada.

\section{Color externo del fruto}

En la Fig. 3 se puede apreciar la evolución del color externo de los frutos de palto cv. 'Hass' a los 0, 4, 8 y 14 días después de concluido el almacenamiento en frío. Allí se observa que al retirarse los frutos de la cámara de conservación no se presentaron diferencias estadísticas entre los tratamientos y el testigo. Sin embargo, a los cuatro días posteriores después de concluido el almacenamiento en frío, se obtuvieron diferencias estadísticas en la coloración de la epidermis; los frutos que recibieron las coberturas de almidón de papa y de almidón de yuca incrementaron su pigmentación perdiéndose sustancialmente el color verde y dando paso a la coloración púrpura típica del cv. 'Hass'. Esta tendencia se hace mucho más notoria a los ocho días de retirados los frutos de almacenaje donde incluso el tratamiento testigo $\mathrm{T}$ y el tratamiento con $\mathrm{CMC}$, en cualquiera de sus concentraciones, también presentaron frutos con coloración, casi similar a lo observado en los tratamientos AY y AP en todas sus concentraciones. Después de 14 días de haberse retirado los frutos del almacenaje en frío, se observa que solo los que fueron tratados con GT en la dosis de $0,6 \%$ reportaron los menores valores de intensidad de coloración púrpura en la piel. Maftoonazad y Ramaswamy (2005) mencionan que la persistencia de la clorofila en la epidermis del palto cv. 'Hass', causante del color verde del fruto, está vinculada a la permanencia y concentración del $\mathrm{CO}_{2}$ en el medio de almacenaje, de tal forma que si esta concentración

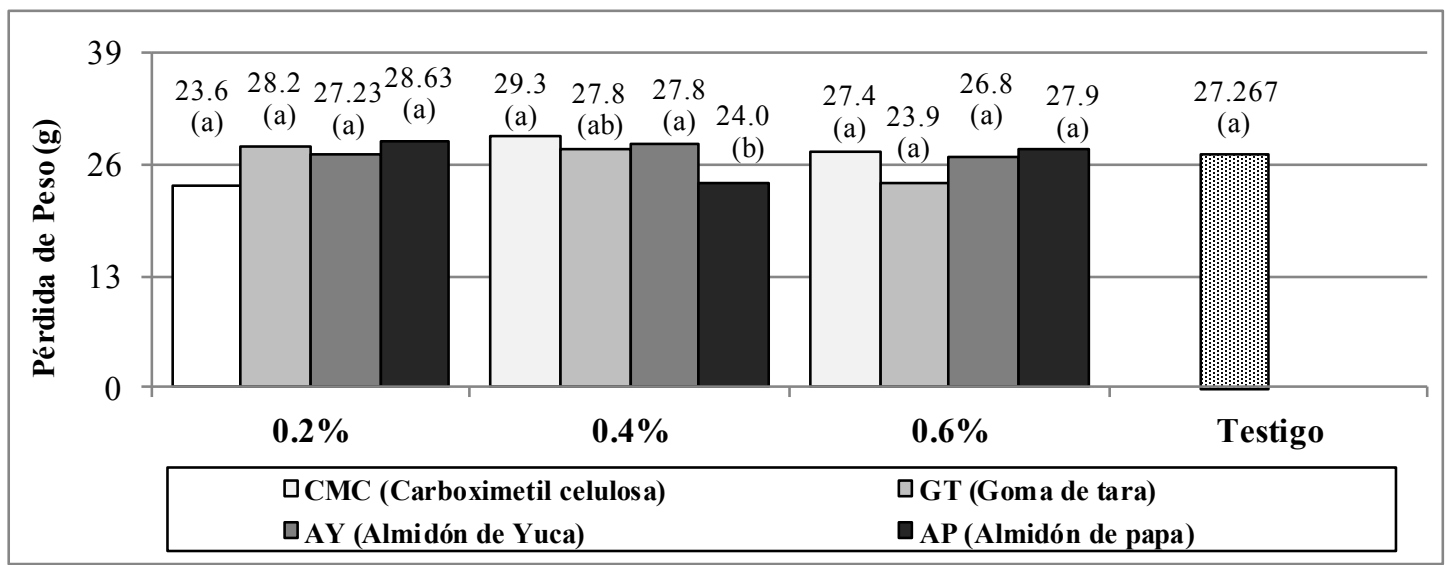

Figura 1. Pérdida de peso en frutos de palto (Persea americana Mill) cv. 'Hass' por efecto de las diferentes concentraciones en cualquiera de las coberturas al concluir el almacenamiento en frío

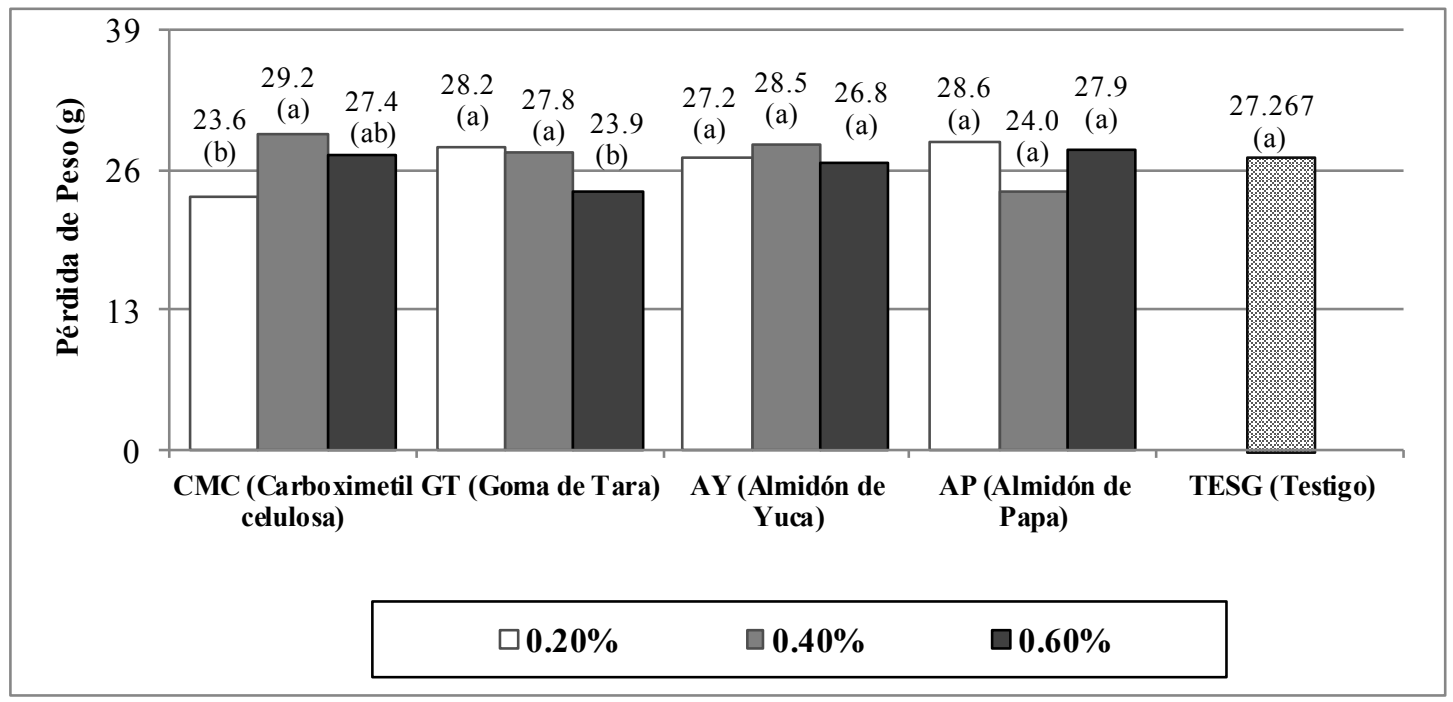

Figura 2. Pérdida de peso en frutos de palto (Persea americana Mill) cv. 'Hass' por efecto de las diferentes coberturas en cualquiera de las concentraciones evaluadas al concluir el almacenamiento en frío 


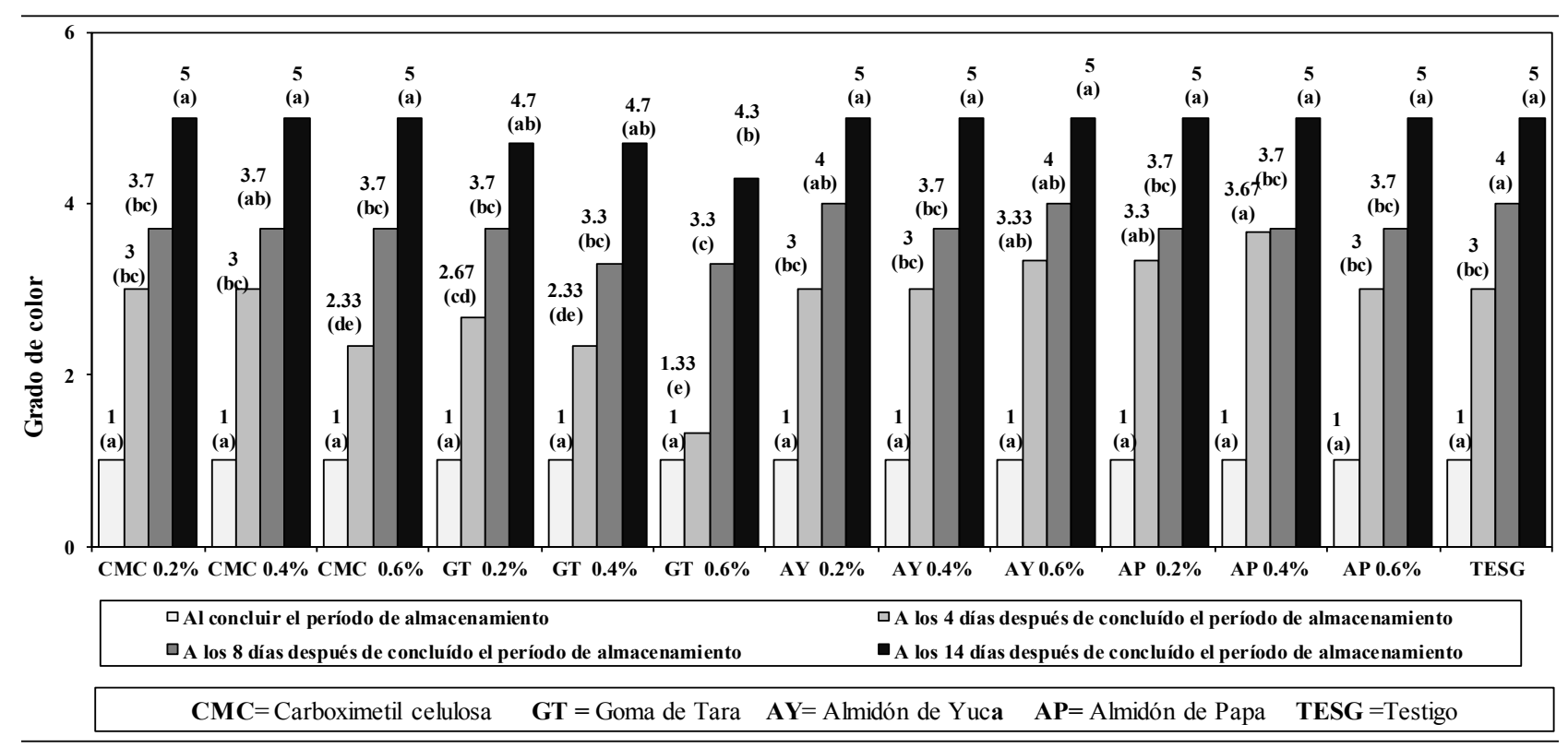

Figura 3. Variación del color externo en frutos de palto (Persea americana Mill) cv. 'Hass' a los 0, 4, 8 y 14 días después de concluido el período de almacenamiento en frío

disminuye, la pérdida de clorofila será mayor dando paso a la aparición de antocianinas. Es así que la concentración y el tipo de cobertura utilizadas sobre los frutos de palto cv. 'Hass' pueden generar un incremento sustancial del $\mathrm{CO}_{2}$ al interior del mismo, tal como lo mencionan Banks et al. (1997). Ello influye sustancialmente en el retraso de la pérdida de clorofila generando una menor aparición de antocianinas en la epidermis de los frutos del palto cv 'Hass', como indican Maftoonazad y Ramaswamy (2005), lo cual se aprecia de manera más notoria con el tratamiento de GT a la dosis de $0,6 \%$.

\section{Dureza}

Los valores de dureza presentados por los frutos de palto cv. 'Hass' al concluir el almacenamiento mostraron diferencias estadísticas por efecto de la interacción tipo coberturaconcentración. El efecto del tipo de cobertura, con relación a cualquiera de las concentraciones utilizadas (Fig. 4), mostró diferencias estadísticas en los frutos que recibieron el tratamiento GT; cuando la concentración fue de 0,6\%, la diferencia fue significativa, mientras que en las coberturas AY y AP la concentración al $0,4 \%$ fue la

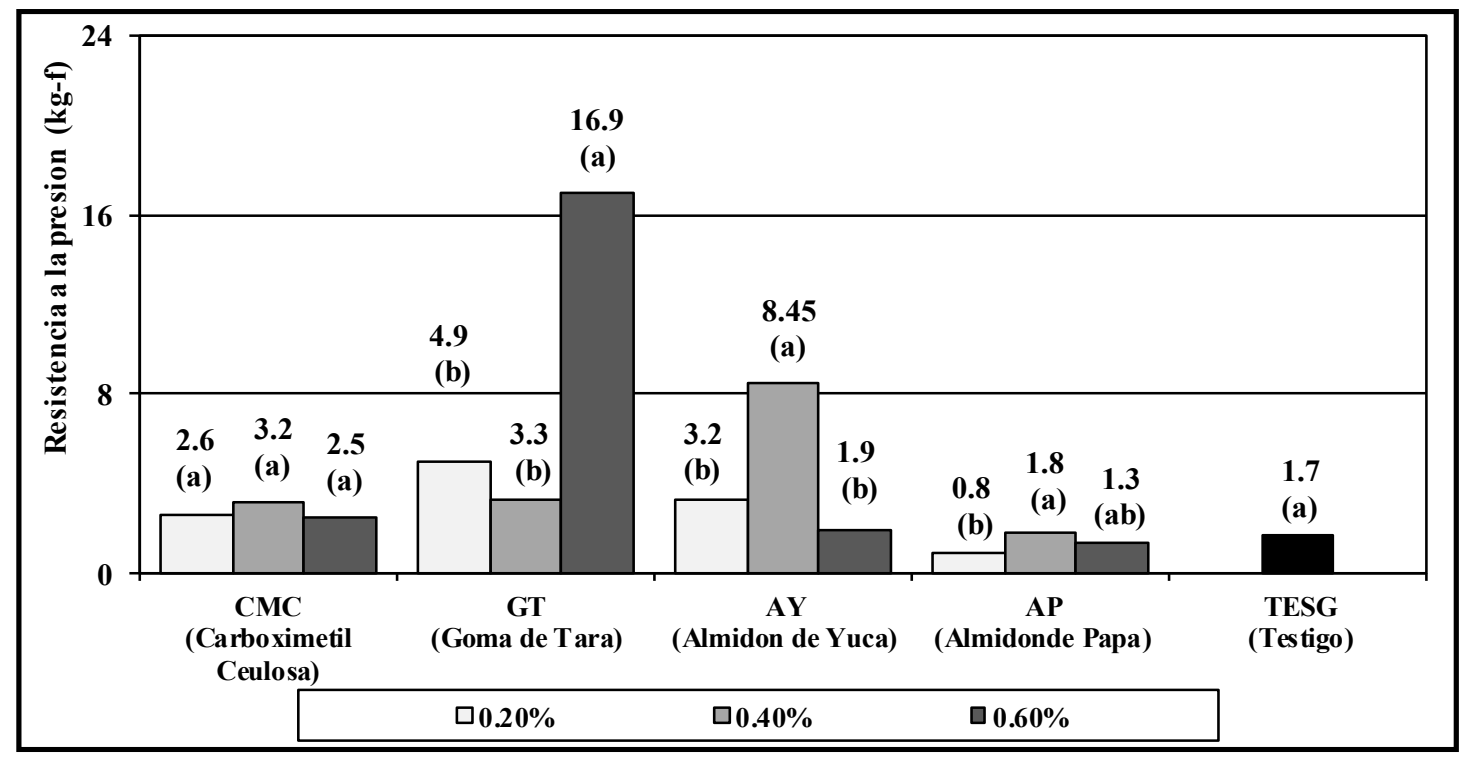

Figura 4. Dureza (kg-f) en frutos de palto (Persea americana Mill) cv. 'Hass' por efecto de las coberturas para las diferentes concentraciones evaluadas al concluir el período de almacenamiento en 
más destacada; sin embargo, de estas coberturas, la de GT al 0,6 \% otorgó el mejor nivel de resistencia. De igual forma, la evaluación de la concentración empleada con relación a cualquiera de las coberturas (Fig. 5) mostró diferencias en la respuesta de la dureza de los frutos de palto cv. 'Hass' y pudo observarse una mejor consistencia de estos conforme la concentración de las coberturas se incrementaba destacando nuevamente el tratamiento de GT al 0,6\% seguido del tratamiento de AY al 0,4\%. El comportamiento observado en la dureza de los frutos al concluir el almacenamiento, se hace más notable 14 días después, dado que decae sustancialmente, tanto por efecto del tipo de cobertura (Fig. 6), como por efecto de las concentraciones (Fig. 7) prevaleciendo una ligera mayor dureza solo en los frutos tratados con GT a la concentración de $0,6 \%$.

El ablandamiento o pérdida de firmeza de los frutos de palta está vinculado principalmente a la degradación de la pared celular en el endocarpio, siendo principalmente las pectinas las más afectadas, debido a la acción de enzimas como la pectinasa y la poligalacturonasa (Aguilar, 2005). Una de las propiedades del uso de coberturas comestibles sobre los órganos vegetales es propiciar cambios importantes en la composición interna de gases, generándose un apreciable incremento de la concentración de $\mathrm{CO}_{2}$ y una caída de los niveles de $\mathrm{O}_{2}$ (Banks et al., 1997; Cisneros y Krochta, 2002). Ello contribuye a una reducción de la actividad enzimática causante de la degradación de las paredes celulares y de la pérdida de firmeza en frutos de palto (Aguilar, 2005), aspecto que es observable en el presente trabajo de investigación, sobre todo con el tipo de cobertura a base de GT aplicada a una concentración del $0,6 \%$.

\section{Pardeado de Pulpa}

El pardeado de la pulpa en frutos de palta cv. 'Hass'

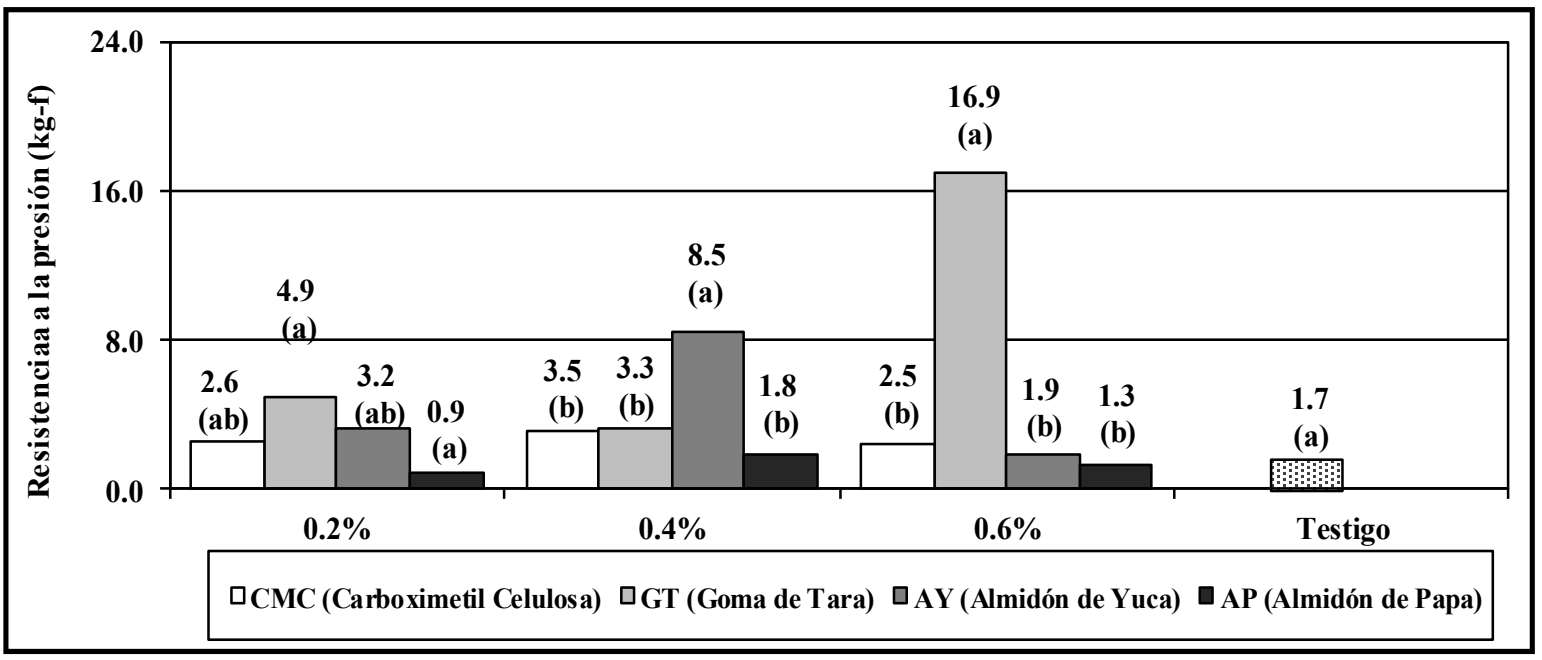

Figura 5. Dureza (kg-f) en frutos de palto (Persea americana Mill) cv. 'Hass' por efecto de las coberturas en relación con las diferentes concentraciones evaluadas al concluir el período de almacenamiento en frío

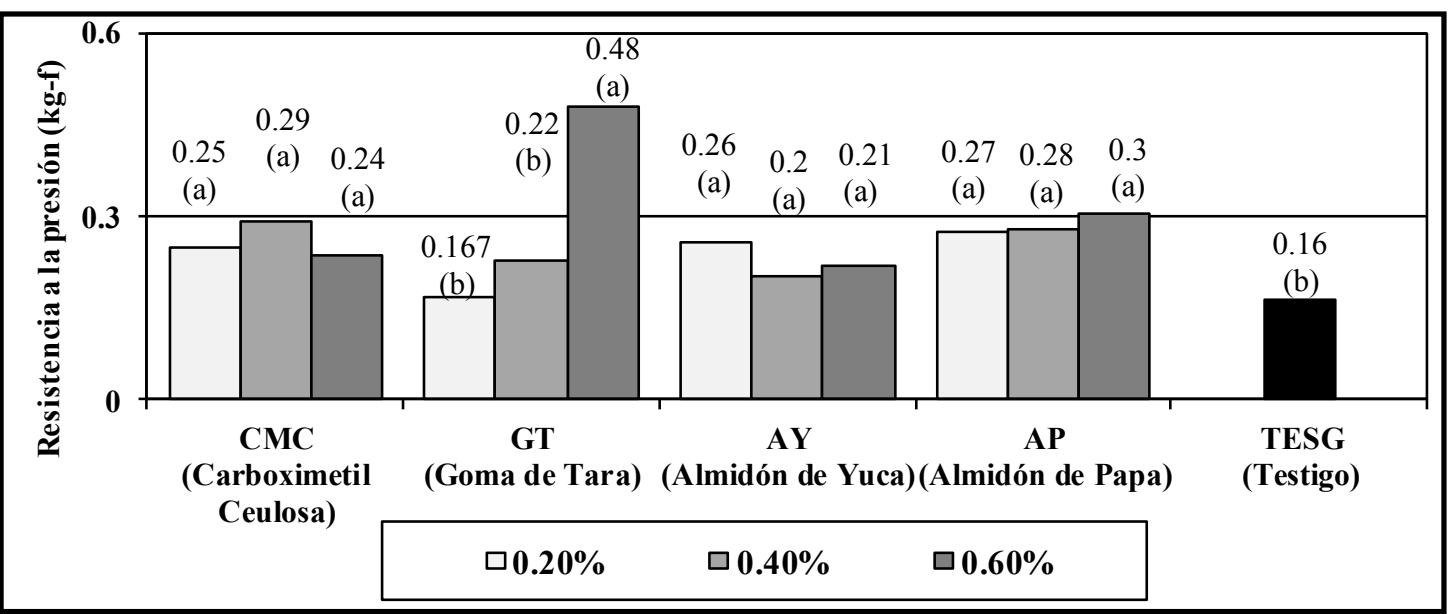

Figura 6. Dureza (kg-f) en frutos de palto (Persea americana Mill) cv. 'Hass' por efecto de las coberturas en relación con las diferentes concentraciones evaluadas a los 14 días después de concluido el almacenamiento en frío 


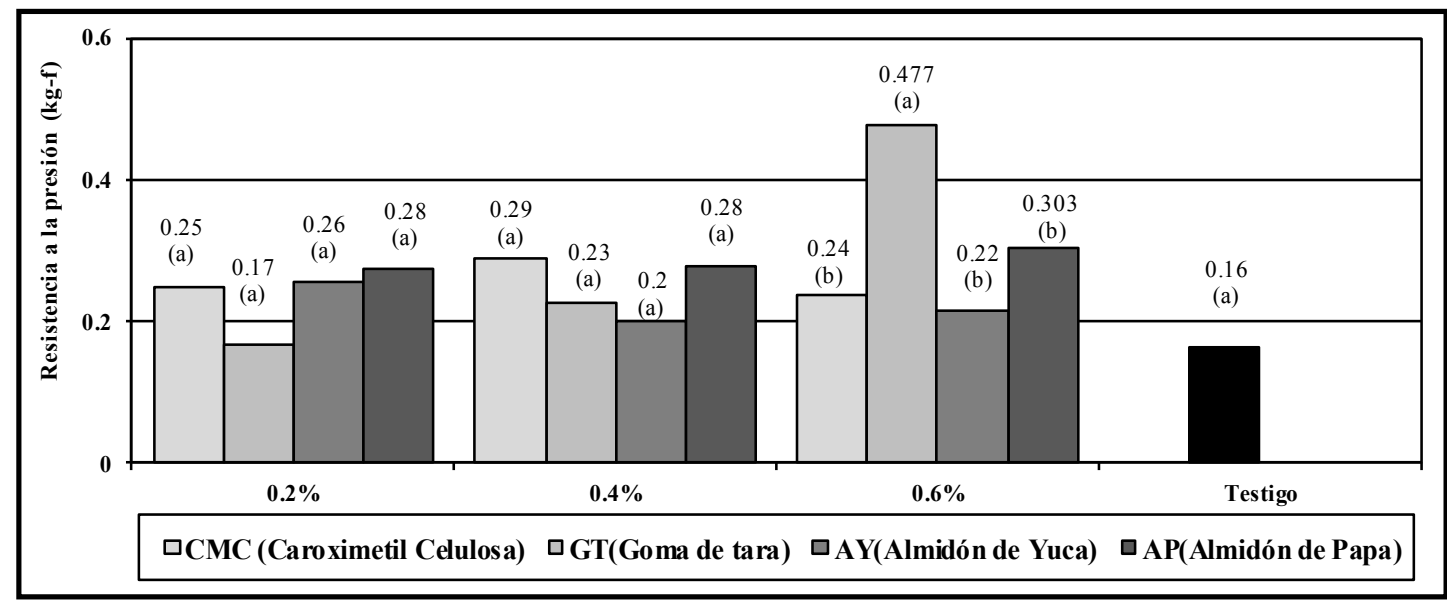

Figura 7. Dureza (kg-f) en frutos de palto (Persea americana Mill) cv. 'Hass' por efecto de las concentraciones en relación con las coberturas evaluadas a los 14 días después de concluido el almacenamiento en frío

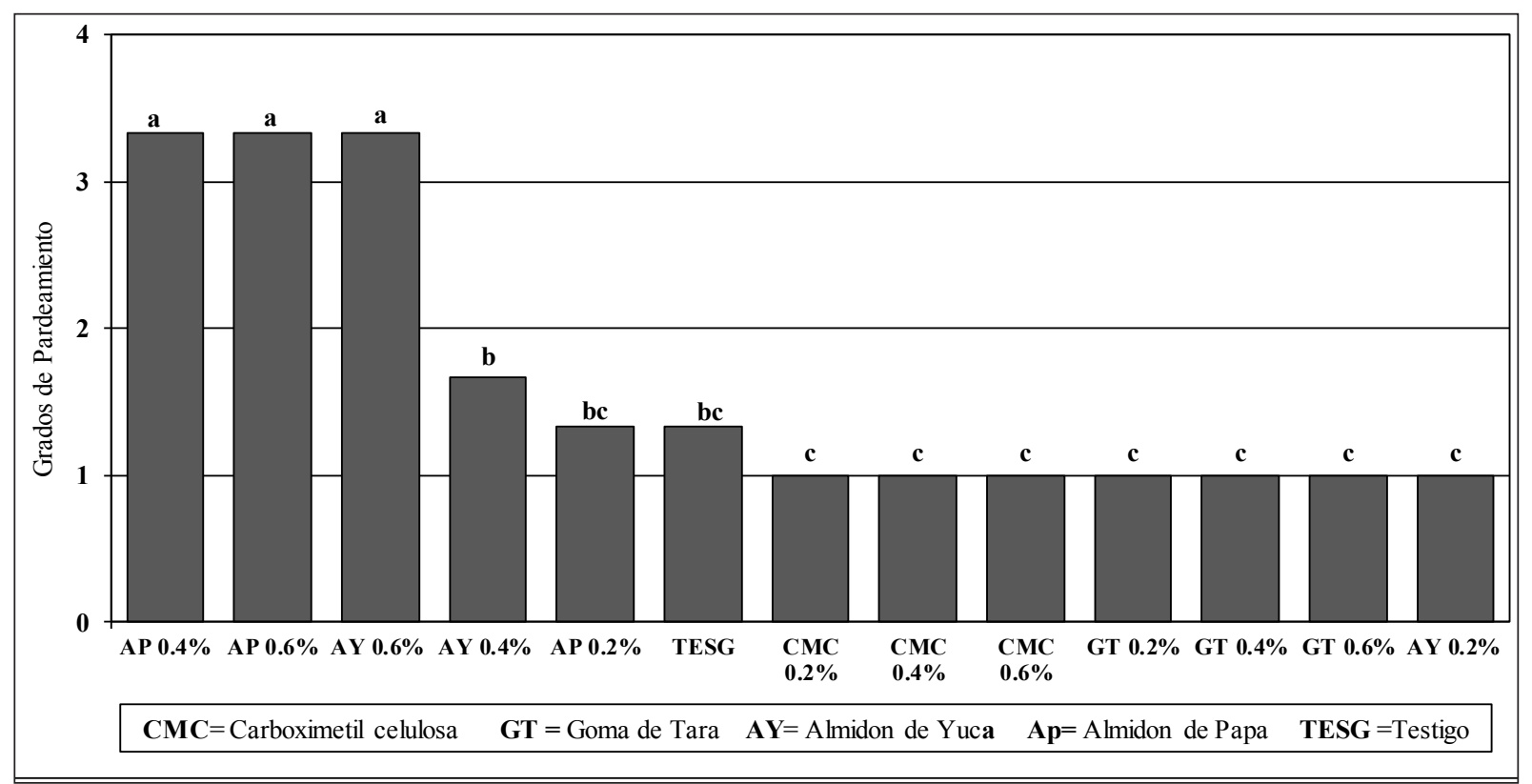

Figura 8. Pardeado de pulpa en frutos de palto (Persea americana Mill) cv. 'Hass' a los 14 días después de concluido el almacenamiento en frío

presentó solo diferencias estadísticas, en la evaluación efectuada a los 14 días después de retirados los frutos del almacenamiento en frío; se mostró más intensamente y de forma similar en los tratamientos en los que prevaleció el almidón como parte de la cobertura, observándose un mayor pardeado interno en aquellos frutos que recibieron coberturas de AP en concentraciones del 0,6 \% y 0,4\%, así como en los tratados con la cobertura AY al 0,6\% $\mathrm{y}$, parcialmente, en aquellos tratados con esta misma cobertura al $0,4 \%$, en tanto su presencia en los frutos que recibieron coberturas a base de CMC y GT en sus diferentes concentraciones (Fig. 8) fue nula. El pardeado de la pulpa en frutos de palta cv. 'Hass' está relacionado con procesos de oxidación bioquímica catalizados por la presencia de enzimas tales como las fenolasas y las polifenoloxidasas, las cuales aparecen conforme las células se vuelven más senescentes o han sufrido daños mecánicos, fisiopatías o cualquier otra acción que determine la desorganización de la integridad celular del fruto u órgano vegetal (Amaya, Tarkus y Domínguez, 2008). En este caso, la exposición de los frutos de palta cv. 'Hass' a una condición de maduración post-almacenaje, determinó que solo algunas de las coberturas ensayadas ofrecieron una mejor protección dilatando el proceso de senescencia de los frutos; esto se evidenció en las coberturas a base de CMC y GT, las cuales generaron una nula aparición del pardeado de pulpa en comparación con las coberturas AY y AP, además del testigo.

\section{Materia seca}

El contenido de materia seca en pulpa evaluada en los frutos, al concluir el almacenamiento en frío, presentó 
diferencias estadísticas entre tratamientos, aspecto que 14 días después no se observó (Tabla 5). A nivel de tratamientos, la cobertura con GT al $0,6 \%$ fue la que reportó los menores valores de materia seca con una clara diferencia en relación con los demás tratamientos. De hecho la cobertura de GT al $0,6 \%$ es posible que haya influido reduciendo la tasa respiratoria e incrementando los niveles internos de $\mathrm{CO}_{2}$ contribuyendo a aminorar el ritmo de senescencia (Banks et al., 1997), retrasando la madurez (Maibee, 1993) y dilatando el ingreso al climaterio de los frutos tratados (Maftoonazad y Ramaswamy, 2005).

\section{Porcentaje de aceite}

Respecto al contenido de aceite, no se obtuvieron diferencias en el análisis estadístico para los distintos tratamientos, tanto al concluir el almacenamiento en frío como a los 14 días después de concluido el almacenamiento (Tabla 6). En general, las coberturas y concentraciones ensayadas no impidieron la acumulación de aceite en la pulpa, ello indica que en general el proceso de maduración y, en todo caso, la síntesis de aceite no se dilató por efecto de los tratamientos, lo cual guarda relación con el hecho de que el proceso de acumulación de aceite durante la maduración de los frutos de palto, tiene una fuerte correlación con el

Tabla 5. Materia seca en la pulpa de frutos de palto (Persea americana Mill) cv. 'Hass' al concluir el almacenamiento en frío y a los 14 días después de concluido el almacenamiento en frío

\begin{tabular}{|c|c|c|c|c|c|c|}
\hline \multirow[b]{3}{*}{ T1: Carboximetil celulosa $0,2 \%$} & \multicolumn{3}{|c|}{$\begin{array}{l}\text { Al concluir el } \\
\text { almacenamiento en frío }\end{array}$} & \multicolumn{3}{|c|}{$\begin{array}{l}\text { A los } 14 \text { días después } \\
\text { de concluido el } \\
\text { almacenamiento en frío }\end{array}$} \\
\hline & \multirow{2}{*}{$\begin{array}{c}\text { Promedio } \\
\text { MS (g) }\end{array}$} & \multicolumn{2}{|c|}{$\begin{array}{c}\% \text { MS } \\
\text { Promedio }\end{array}$} & \multirow{2}{*}{$\begin{array}{c}\begin{array}{c}\text { Promedio } \\
\text { MS (g) }\end{array} \\
12,63\end{array}$} & \multicolumn{2}{|c|}{$\begin{array}{c}\% \mathrm{MS} \\
\text { Promedio }\end{array}$} \\
\hline & & 25,6 & $\mathrm{AB}$ & & 25,3 & A \\
\hline T2: Carboximetil celulosa $0,4 \%$ & 12,67 & 25,3 & $\mathrm{AB}$ & 13,47 & 26,9 & A \\
\hline T3: Carboximetil celulosa $0,6 \%$ & 12,13 & 24,3 & $\mathrm{AB}$ & 13,07 & 26,1 & A \\
\hline T4: Goma de Tara $0,2 \%$ & 12,53 & 25,1 & $\mathrm{AB}$ & 12,60 & 25,2 & A \\
\hline T5: Goma de Tara $0,4 \%$ & 12,60 & 252 & $\mathrm{AB}$ & 12,83 & 25,7 & A \\
\hline T6: Goma de Tara $0,6 \%$ & 10,43 & 20,9 & $\mathrm{~B}$ & 11,67 & 233 & A \\
\hline T 7: Almidón de Yuca $0,2 \%$ & 12,70 & 25,4 & $\mathrm{AB}$ & 13,03 & 26,1 & A \\
\hline T 8: Almidón de Yuca 0,4\% & 10,97 & 21,9 & $\mathrm{AB}$ & 13,40 & 26,8 & A \\
\hline T9: Almidón de Yuca 0,6\% & 12,80 & 25,6 & $\mathrm{AB}$ & 12,13 & 24,3 & A \\
\hline T10: Almidón de Papa $0,2 \%$ & 13,77 & 27,5 & A & 12,97 & 25,9 & A \\
\hline T11: Almidón de Papa 0,4\% & 13,43 & 26,9 & A & 12,63 & 25,3 & A \\
\hline T12: Almidón de Papa $0,6 \%$ & 13,53 & 27,1 & A & 12,27 & 24,5 & A \\
\hline T13: Tratamiento “0” & 12,37 & 24,7 & $\mathrm{AB}$ & 14,60 & 29,3 & A \\
\hline
\end{tabular}

Tabla 6. Porcentaje de aceite en frutos de palto (Persea americana Mill) cv.'Hass' al concluir el almacenamiento en frío y a los 14 días después de concluido el almacenamiento en frío

\begin{tabular}{|c|c|c|c|c|}
\hline Tratamiento & \multicolumn{2}{|c|}{$\begin{array}{c}\text { Al concluir el } \\
\text { almacenamiento en frío } \\
\text { \% Promedio de Contenido } \\
\text { de Aceite } \\
\end{array}$} & \multicolumn{2}{|c|}{$\begin{array}{c}\text { A los } 14 \text { días después } \\
\text { de concluido el } \\
\text { almacenamiento en frío } \\
\text { \% Promedio de Contenido } \\
\text { de Aceite }\end{array}$} \\
\hline T1: Carboximetil celulosa $0,2 \%$ & 13,033 & A & 14,9610 & A \\
\hline T2: Carboximetil celulosa $04 \%$ & 13,467 & A & 15,7348 & $\mathrm{~A}$ \\
\hline T3: Carboximetil celulosa $0,6 \%$ & 13,067 & A & 15,4022 & $\mathrm{~A}$ \\
\hline T4: Goma de Tara $0,2 \%$ & 12,833 & A & 14,7813 & $\mathrm{~A}$ \\
\hline T5: Goma de Tara $0,4 \%$ & 11,667 & A & 15,0599 & A \\
\hline T6: Goma de Tara $0,6 \%$ & 10,100 & A & 13,4351 & A \\
\hline T 7: Almidón de Yuca $0,2 \%$ & 12,633 & A & 15,6196 & $\mathrm{~A}$ \\
\hline T 8: Almidón de Yuca 0,4\% & 10,967 & A & 15,8302 & $\mathrm{~A}$ \\
\hline T9: Almidón de Yuca 0,6\% & 12,800 & A & 14,4267 & A \\
\hline T10: Almidón de Papa $0,2 \%$ & 14,600 & A & 15,9847 & A \\
\hline T11: Almidón de Papa 0,4\% & 12,633 & A & 15,2793 & $\mathrm{~A}$ \\
\hline T12: Almidón de Papa 0,6\% & 13,800 & A & 14,5750 & $\mathrm{~A}$ \\
\hline T13: Tratamiento " 0 " & 13,300 & A & 15,9919 & A \\
\hline Letras iguales no presentan diferencias $(\mathrm{p}=0,05)$ & CV: 17,02 & & CV: 6,92 & \\
\hline
\end{tabular}




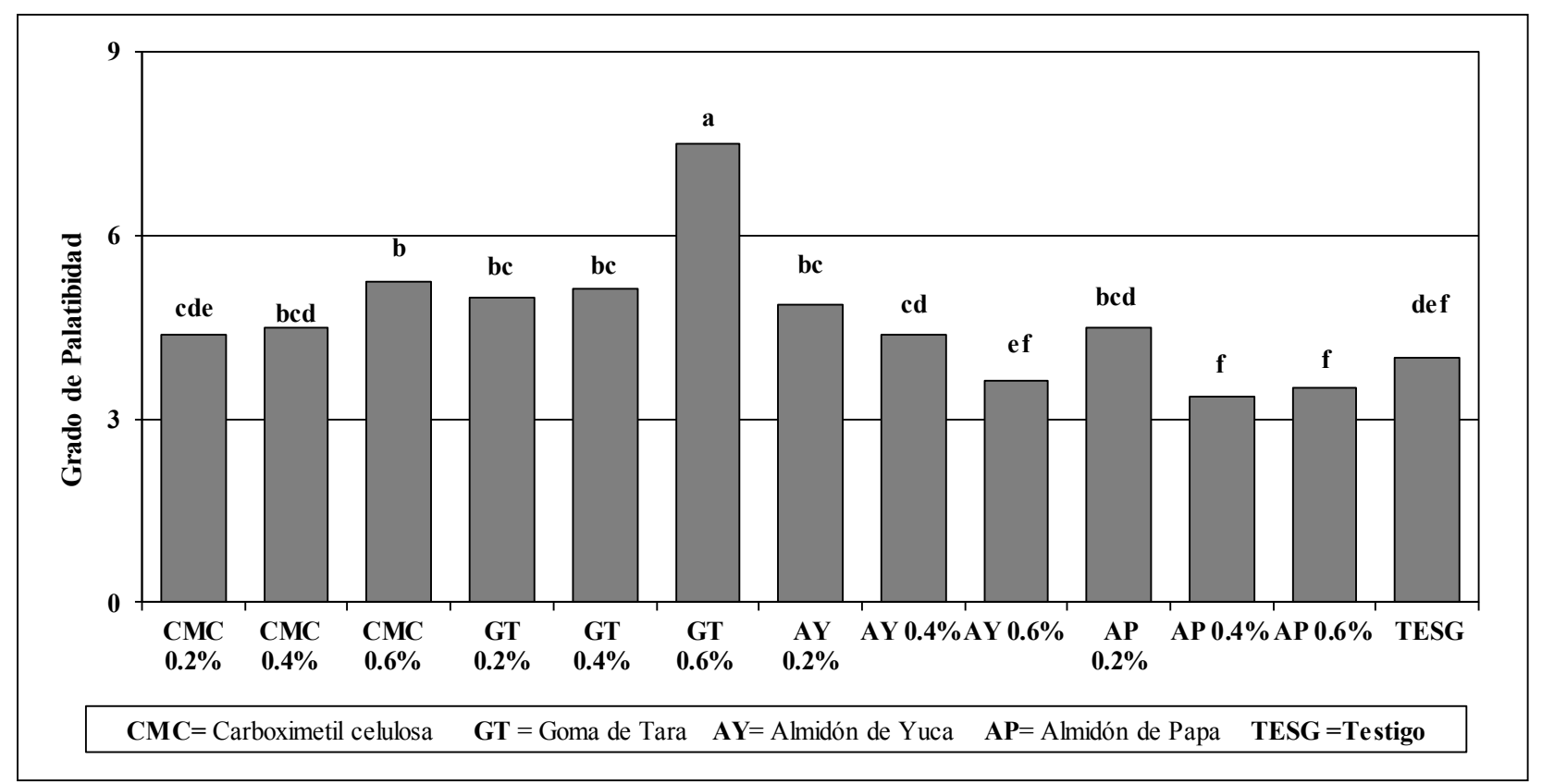

Figura 9. Palatabilidad en frutos de palto (Persea americana Mill) cv. 'Hass' a los 14 días después de concluido el almacenamiento en frío

incremento de materia seca (Parodi et al., 2007; Kassim et al., 2013) y juega un papel muy limitado en la fisiología y comportamiento poscosecha de estos frutos (Blakey et al., Bower, 2011).

\section{Palatabilidad}

La evaluación del nivel de aceptación de los frutos de palto cv. 'Hass', realizada a los 14 días después de concluido el almacenamiento en frío, presentó notorias diferencias estadísticas (Fig. 9). Al comparar los tratamientos, se observa que la cobertura GT al $0,6 \%$ destacó significativamente recibiendo una calificación entre "me gusta medianamente" (puntaje 7) y "me gusta mucho" (puntaje 8). Esta mejor aceptación por parte del panel degustador fue menos apreciada en los tratamientos con GT a las dosis de $0,2 \%$ y $0,4 \%$, asimismo con los tratamientos de $\mathrm{CMC}$ al $0,6 \%$ y de $\mathrm{AY}$ al $0,2 \%$. Los demás tratamientos generaron rechazo por parte del panel degustador.

Es claro que las coberturas evitan la senescencia acelerada de los frutos de palto, tal como lo indican Banks et al., 1997. Por lo que la concentración de componentes acetaldehídos y cetonas es menos intensa, evitando con ello la pérdida del sabor en los frutos de palto (Kassim et al., 2013). En este caso, el tratamiento GT al 0,6 \% logra reducir de manera más efectiva la síntesis de estos compuestos proporcionando una mejor aceptación en la prueba de palatabilidad.

\section{Conclusiones}

La evaluación de los frutos de palto cv. 'Hass', al concluir el almacenamiento en frío, mostró que la cobertura de GT al 0,6 \% otorgó una mayor dureza y mantuvo menores valores de materia seca en comparación con los demás tratamientos.
La evaluación de los frutos de palto cv. 'Hass', a los 14 días después de concluir el almacenaje en frío, mostró que la cobertura GT al 0,6 \% generó un retraso significativo en la aparición del color púrpura de la epidermis, mantuvo más firmeza y mejor palatabilidad.

No se observó pardeado interno en los frutos de palto cv. 'Hass', a los 14 días después de concluido el almacenaje en frío, cuando estos fueron tratados con las coberturas a base de CMC y GT en sus diferentes dosis, así como la de AY al 0,2\%.

En general, del total de coberturas y concentraciones ensayadas, el tratamiento con GT al 0,6 \% mostró la mejor respuesta logrando reducir el deterioro o senescencia de los frutos de palto cv. 'Hass' sometidos a almacenaje en frío.

\section{Literatura citada}

Aguilar, M.A. 2005. Propiedades físicas y mecánicas de películas biodegradables y su empleo en el recubrimiento de frutos de aguacate. (Tesis Maestro Tecn. Avan.). Instituto Politécnico Nacional-CICATA. México D.F.

Amaya, P.E.; Tarkus, P.R. y Domínguez, M.M. 2008. Extracción y caracterización cinética de la enzima polifenoloxidasa del aguacate (Persea americana Mill.) variedad 'Hass'. Revista de la Facultad de Ingeniería Química, Univ. Aut. de Yucatán. Vol. 47, 10-16.

Association of Official Analytical Chemist International [AOAC]. 1990. Official Methods of Analysis of AOAC International. (15ta. ed.) Ed.: K. Helrich.

Baldwin, E.A. 1994. Edible coatings for fresh fruits and vegetables: past, present, and future. In: Edible Coatings and Films to Improve Food Quality. Krochta, J.M., Baldwin, E.A., Nisperos Carriedo, M.O. (eds.). Technomic Publishing, Lancaster Basel. 
Banks, N.; Johnston, J.; Watson, A.; Kingley, A. y Mackay, B. 1997. Coating to enhance fruit life. Proceedings from conference '97: Searching for Quality. Joint Meeting of the Australian Avocado Grower's Federation, Inc. and NZ Avocado Growers Association. 23-26 September. JG. Cuttings eds. 46-54 p.

Blakey, R.; Tesfay, S.; Bertling, I. y Bower, J. 2011. The relationships between sugars, protein and oil of 'Hass' avocado fruit during ripening. In: Management of Avocado Postharvest Physiology Submitted in partial fulfilment of the requirements (degree of $\mathrm{phD}$ in Agriculture-Discipline) of Horticultural Science School of Agricultural Sciences and Agribusiness Faculty of Science and Agriculture University. South Africa: KwaZulu-NatalPietermaritzburg, pp. 75-94.

Cancino, C. 2007. Efecto del uso de dos coberturas en la reducción de daños por frío en palta cv. 'Hass'. Taller de Licenciatura. Quillota, CL, Facultad de Agronomía. Pontificia Universidad Católica de Valparaíso.

Cisneros, Z. y Krochta, L. 2002. Internal modified atmospheres of coated fresh fruits and vegetables: understanding relative humidity effects. J. Food Sci., 67(8): 2792-2797.

Corzo, J.A. 2005. Estadística no paramétrica: métodos basados en rangos. (1 ra ed.) Facultad de Ciencias. Universidad Nacional de Bogotá.

Kassim, A.; Workneh, T.S. y Bezuidenhout, C.N. 2013. A review on postharvest handling of avocado fruit. Afr. $J$. Agric. Res., Vol 8(21): 2385-2402.

Maftoonazad, N. y Ramaswamy, H. 2005. Postharvest shelf-life extension of avocados using methyl cellulosebased coating. Swiss Society of Food Science and Technology. Elsevier Ltd. LWT, 38: 617-624.

Maibee, A.L. 1993. Efecto de una cobertura de ceras naturales y éster de sacarosa, sobre el comportamiento en almacenaje refrigerado de paltas (Persea americana Mill.) cv. 'Fuerte' en distintos niveles de madurez. (Tesis Ing. Agro.). Facultad de Agronomía. Área de Horticultura. Universidad Católica de Valparaíso. Chile.

Pantástico, E.B. 1984. Fisiología de la Postrecolección, Manejo y Utilización de Frutas y Hortalizas. México: Ed. Continental S.A.

Parodi, G. y Lerner, M. 1995. Ensayo sobre almacenamiento en frío de palto 'Hass'. Proc. Interamer. Soc. Trop. Hort, 39: 86-93.

Parodi, G.; Sanchez, M. y Daga, W. 2007. Correlación del contenido de aceite, materia seca y humedad de pulpa como indicadores de cosecha en frutos de palto (Persea americana Mill) var. 'Hass' cultivada bajo condiciones de dos localidades en Chincha-Perú. En Actas VI Congreso Mundial del Aguacate. Viña Del Mar, Chile.

Royal Horticulture Society, UK [RHS]. 2001. Color Chart (RHSCC). (4ta. ed.). London, Great Britain (4 fan colored charts).

Wills, R.; Lee, T.; Mc Glasson, W.B.; Hall, E.G. y Graham, D. 1984. Fisiología y manipulación de frutas y hortalizas postrecolección. Acribia, Zaragoza, España. 